\title{
Identification of aphidicolin-induced fragile sites in domestic pig chromosomes
}

\author{
PK Riggs, CL Chrisman $\dagger$ \\ Purdue University, Cytogenetics Laboratories, Departement of Animal Sciences, \\ West Lafayette, IN 47907, USA
}

(Proceedings of the 9th European Colloquium on Cytogenetics of Domestic Animals; Toulouse-Auzeville, 10-13 July 1990)

chromosome / fragile sites / pig

\section{INTRODUCTION}

Fragile sites are specific chromosomal loci prone to breakage which are observed as gaps, breaks or rearrangements under appropriate in vitro culture conditions. More than 100 fragile sites have been identified in human chromosomes and classified according to their mode of induction and population frequency (Sutherland and Ledbetter, 1989). All fragile sites, by definition, are inducible in culture, and induction results in their expression or elevates the proportion of cells in which fragile sites are observed if they are 'spontaneously' expressed (Sutherland and Hecht, 1985). Fragile sites are also identified as rare (occurring in less than $1 \%$ of the population) or common.

One useful fragile site-inducing agent is aphidicolin (APC). This chemical is a selective inhibitor of DNA polymerase- $\alpha$, the major eukaryotic polymerase which adds deoxynucleotide monophosphates to the $3^{\prime}$-end of a DNA primer, during both replicative and excision repair DNA synthesis (Fry and Loeb, 1986). At low concentrations $(0.2 \mu \mathrm{M})$, APC has been used to induce fragile sites in human lymphocyte cultures without significantly decreasing mitotic yield (Glover et al, 1984).

The significance of fragile sites remains unclear, although numerous hypotheses have been suggested linking fragile sites to cancer chromosome breakpoints and protooncogene locations (LeBeau, 1988), reciprocal translocation breakpoints (Riggs and Chrisman, 1989), chromosome rearrangements resulting in spontaneous abortion and infertility (Schlegelberger et al, 1989) and evolutionary preservation of syntenic groups (Threadgill and Womack, 1989). Chromosome aberrations, especially reciprocal translocations, are of economic importance in the domestic pig

† Deceased November 22, 1990. 
because they usually result in significantly reduced fertility (reviewed by Popescu et al, 1984).

Although extensively documented in human chromosomes, the study of fragile sites has been neglected in domestic animals. Since fragile sites are distributed nonrandomly, and it has been suggested that reciprocal translocations are distributed non-randomly across the pig genome (Fries and Stranzinger, 1982), this investigation was undertaken to study common fragile sites in pig chromosomes. The initial objectives of the project were 3 -fold: 1 ) establish protocols for studying fragile sites in chromosomes of domestic animals, specifically the pig; 2) identify band locations of fragile sites in pig chromosomes; 3 ) determine whether a correlation exists between common fragile sitc locations and reciprocal translocation breakpoints.

\section{MATERIALS AND METHODS}

Peripheral blood was collected via the anterior vena cavae of 2 male and 2 female purebred Duroc pigs, and 7 male and 8 female 3- or 4-way crossbred pigs (Yorkshire $\times$ Landrace $\times$ Hampshire ; Yorkshire $\times$ Chester White $\times$ Hamsphire ; Yorkshire $\times$ Landrace $\times$ Hampshire $\times$ Duroc $;$ Yorkshire $\times$ Chester White

$\times$ Hampshire $\times$ Duroc $\quad$ ). Standard cultures were established with RPMI-1640 (Gibco), 10\% fetal bovine serum (Gibco), 2\% phytohemagglutinin-M (Gibco), 1\% 1-glutamine, $0.3 \%$ sodium heparin, $0.5 \%$ gentamicin sulfate and $5 \%$ whole blood. Cultures were grown in $5 \% \mathrm{CO}_{2}$ in air at $37^{\circ} \mathrm{C}$ for $64.5 \mathrm{~h}$. Aphidicolin (Sigma) was dissolved in ethanol and diluted with saline. Cultures received APC or the carrier solution $24 \mathrm{~h}$ prior to harvest.

To establish dose-response curves and determine the optimal APC concentration for study, 50 solid-stained metaphases per culture were scored for gaps, breaks and rearrangements in both crossbred and Duroc pigs. Chromosome preparations from controls and cultures that received $0.2 \mu \mathrm{M}$ APC were trypsin-Giemsa (GTG-) banded, and band locations according to the international nomenclature (Committee for the Standardized Karyotype of the Domestic Pig, 1988) were assigned to observed gaps, breaks and rearrangements.

\section{RESULTS AND DISCUSSION}

Aphidicolin induced gaps, breaks and rearrangements in pig chromosomes when added to lymphocyte cultures $24 \mathrm{~h}$ before harvest. Induction of chromosome aberrations was dependent upon the APC concentration, and response was similar to that demonstrated for human chromosomes by Glover et al (1984). Gaps, breaks and rearrangements induced by $0.2 \mu \mathrm{M} \mathrm{APC}$. were analyzed from 345 metaphase plates from 7 individuals. A total of 345 aberrations were observed at 94 different band locations. Only 3 breaks were observed in 350 metaphases from control cultures.

So-called common fragile sites were identified by $\chi^{2}$ analysis. Based on a 287band, standard GTG karyotype, and assuming each band had an equal probability of breakage, the expected number of breaks from the 345 aberrations induced by $0.2 \mu \mathrm{M}$ APC was 1.20 breaks/band. $\chi^{2}$ analysis indicated that any band with 4 
or more breakage events was significantly damaged $\left(\chi^{2}, 1 d f \geq 4.41\right.$ with Yates' correction; $P<0.05)$. Band locations of fragile sites with 4 or more breakage events are listed in table I. Of 94 different breakpoints, 21 locations were significantly damaged.

Table I. Aphidicolin-induced fragile sites in domestic pig chromosomes.

\begin{tabular}{cl}
\hline No of breaks & \multicolumn{1}{c}{ Band locations } \\
\hline $4^{\mathrm{b}}$ & $4 \mathrm{q} 15,6 \mathrm{p} 15,13 \mathrm{q} 33, \mathrm{Xp} 24$ \\
$5^{\mathrm{c}}$ & $1 \mathrm{q} 21.1,11 \mathrm{p} 13,13 \mathrm{q} 41,17 \mathrm{q} 21, \mathrm{Xq} 24$ \\
$6^{\mathrm{d}}$ & $1 \mathrm{p} 21,1 \mathrm{q} 17,4 \mathrm{q} 23,6 \mathrm{q} 31$ \\
$7^{\mathrm{d}}$ & $4 \mathrm{p} 15,13 \mathrm{q} 21$ \\
$11^{\mathrm{d}}$ & $\mathrm{Xq} 26$ \\
$14^{\mathrm{d}}$ & $1 \mathrm{p} 25, \mathrm{Xq} 22$ \\
$21^{\mathrm{d}}$ & $1 \mathrm{p} 23$ \\
$31^{\mathrm{d}}$ & $10 \mathrm{p} 15$ \\
$47^{\mathrm{d}}$ & $4 \mathrm{q} 25$ \\
\hline
\end{tabular}

a Number of breaks observed at specific band locations in 345 metaphases from 7 individuals.

${ }^{\mathrm{b}} P<0.05 ;{ }^{\mathrm{c}} P<0.005 ;{ }^{\mathrm{d}} P<0.001$.

Breakpoints of 29 reciprocal translocations (43 different breakpoints) reported in the literature were compared to APC-induced chomosome breakpoints. Twentynine locations were identified as both translocation and APC-induced breakpoints. $\chi^{2}$ analysis indicated that $\mathrm{APC}$-induced breakage and reciprocal translocation breakpoints were not independent $\left(\chi^{2}, 12 d f=57.30 ; P<0.001\right)$.

The primary objective of this study was to establish protocols for studying fragile sites in pig chromosomes and identify the common APC-induced fragile sites in the pig genome. At $0.2 \mu \mathrm{M}$ concentration, as in previous human fragile site studies, APC was useful for inducing non-random gaps, breaks and rearrangements, $i e$, fragile sites. Twenty-one fragile sites located on 8 chromosomes $(1,4,6,10,11,13$, $17, \mathrm{X})$ were identified. The relationship of fragile sites to reciprocal translocation breakpoints was also examined. Breakage was induced in 29 bands which had been previously identified as translocation breakpoints. Preliminary analysis indicated that the two events were not independent. Stronger conclusions cannot be drawn at this time, however, because no data are available concerning the actual population frequency of de novo (or environmentally induced) translocation incidents. Also, translocations which arise at some fragile sites may result in inappropriate gene expression and be lethal. These translocations would probably never be observed.

This study was designed as a preliminary examination of chromosomal fragile sites in domestic animals. A better understanding of the nature of fragile sites should provide insight for clinical and research investigations on mechanisms of environmental mutagenesis and in vivo induction of chromosome aberrations associated with reproductive problems. 


\section{REFERENCES}

Committee for the Standardized Karyotype of the Domestic Pig (1988) Standard karyotype of the domestic pig. Hereditas 109, 151-157

Fries R, Stranzinger G (1982) Chromosomal mutations in pigs derived from X-irradiated semen. Cytogenet Cell Genet 34, 55-66

Fry M, Loeb LA (1986) Animal Cell DNA Polymerases. CRC Press, Boca Raton, FL

Glover TW, Berger C, Coyle J, Echo B (1984) DNA polymerase- $\alpha$ inhibition by aphidicolin induces gaps and breaks at common fragile sites in human chromosomes. Hum Genet 67, 136-142

LeBeau MM (1988) Chromosomal fragile sites and cancer-specific breakpoints - a moderating viewpoint. Cancer Genet Cytogenet 31, 55-61

Popescu CP, Bonneau M, Tixier M, Bahri I, Boscher J (1984) Reciprocal translocations in pigs. $J$ Hered $75,448-452$

Riggs PK, Chrisman CL (1989) Preliminary analyses of aphidicolin-induced fragile sites in pig chromosomes. Sixth North American Colloquium on Cytogenetics of Domestic Animals. West Lafayette, Indiana, abstr 4

Schlegelberger B, Gripp K, Grote W (1989) Common fragile sites in couples with recurrent spontaneous abortions. Am J Med Genet 32, 45-51

Sutherland GR, Hecht F (1985) Fragile Sites on Human Chromosomes. Oxford University Press, New York

Sutherland GR, Ledbetter DH (1989) Report of the Committee on Fragile Sites, Human Gene Mapping 10. Cytogenet Cell Genet 51, 324-332

Threadgill DW, Womack JE (1989) Syntenic assignment of HSA 9 and HSA 12 homologs in the bovine. Preliminary evidence for the role of fragile sites in mammalian genome evolution. Cytogenet Cell Genet 51, 1091 (abstr a2664, HGM 10) 